\title{
Les constructions allocentrées : une description sémantico-énonciative ${ }^{1}$
}

\author{
Georgeta Cislaru \\ SYLED-CEDISCOR, Université Sorbonne nouvelle Paris 3 \\ gcislaru@univ-paris3.fr
}

Ce travail s'inscrit dans le champ plus large des questionnements portant sur les notions d'intersubjectivité, d'échange et de communication empathique (cf. Forest, Kuno, Zahavi, Rimé, etc.), le but étant de vérifier la nature intersubjective du langage et d'identifier les marques linguistiques signifiant le lien entre Soi et l'Autre. Nous allons définir l'intersubjectivité, en partant d'une analyse morphologique du terme, comme un rapport de solidarité ou de positionnement réciproque entre des sujets parlants, linguistiquement ou discursivement marqué. Communication et action sont envisagées comme impliquant nécessairement deux pôles, le sujet parlant pouvant prendre - ou, au contraire, éviter de le faire - l'Autre (interlocuteur ou, éventuellement, tiers) comme point de repère ${ }^{2}$, comme garant ou comme actant.

Le marquage linguistico-discursif des rapports qui s'établissent entre sujets parlants a pu être abordé sous plusieurs angles dans la littérature (voir Kuno 1987, Forest 1999) : énonciation (rapports entre je et $t u$, notion de point de vue, utilisation du style indirect libre), lexique (choix lexical celui-ci vs celui-là, le frère de Marie vs Pierre), pragmatique (théorie des actes du langage), syntaxe (impossibilités de reprise anaphorique : * the alcoholic's wife $_{i}$ killed her $_{i}$ husband; emploi de l'imparfait pour parler d'une personne vivante ; J'ai le bras qui me fait mal vs J'ai mal au bras). Il est supposé dans ces travaux que certaines unités des langues ont des affinités avec certains schèmes de lecture empathique, ou, plus généralement, d'intersubjectivation. Cette hypothèse peut s'appliquer avec profit à l'objet qui est le nôtre : l'expression des émotions en français.

L'étude que nous proposons ici s'inscrit dans le cadre d'une sémantique énonciative et porte sur des constructions syntaxiques ayant un format bien déterminé : N1humain+expression émotionnelle+pour + N2humain ${ }^{3}$. Au niveau grammatical, elles concernent aussi bien des noms que des verbes ou des adjectifs, comme le montrent les exemples 1-3.

Ces constructions sont attestées dès la première moitié du $17^{\text {ème }}$ siècle (cf. 1, sondage Frantext).

(1) J'ay grand peur pour vous que l'Antechrist ne naisse bien tost en vostre Diocese (BALZAC (GUEZ DE) Jean-Louis, Les Premières lettres : t. 2 (1627), 1627, p. 54)

(2) $[\ldots]$ cette petite flamme-tu sais, ta petite flamme ? - depuis longtemps, je ne la voyais plus s'allumer ; depuis un an, peut-être... enfin, je me réjouis pour toi... vous êtes restés ensemble moins d'un quart d'heure... (MAURIAC François, Les Mal Aimés, 1945, p. 239, ACTE III scène 5)

(3) Je me sens très triste pour elle. Je ressens ce regret, cette tristesse : elle est privée de ses enfants. (DUPEREY Annie, Je vous écris, 1993, p. 137)

Nous les appellerons constructions allocentrées, compte tenu du lien de solidarité qui semble s'établir entre le N1 et le N2 et qui s'inscrit dans une dynamique intersubjective. Mais ces constructions soulèvent la question de l'attribution de l'émotion et de la causalité, en problématisant ainsi le statut sémanticosyntaxique de $\mathrm{N} 1$ et de N2 : qui est de fait le siège de l'émotion? N2 est-il le stimulus ou la cause de l'émotion? Il s'agit donc de mettre en regard le schéma sémantique de ces constructions et les schèmes d'intersubjectivation.

Après quelques remarques liminaires visant à situer la problématique, nous commencerons par départager les constructions allocentrées des structures syntaxiques similaires en proposant un premier cadre interprétatif. Nous observerons ensuite la répartition des rôles sémantiques (siège, stimulus) en prenant 
comme point de départ le modèle sémantico-syntaxique de Bresson \& Dobrovol'skiy $X$ a peur de $Y / \grave{a}$ cause de Y (Bresson \& Dobrovol'skiy 1995). Compte tenu de la dissymétrie d'emploi selon que l'on parle ou non à la $1^{\text {ère }}$ personne, et du fait que seul l'usage de la $1^{\text {ère }}$ personne rend compte de l'expression linguistique des émotions (cf. Kerbrat-Orrecchioni $2000: 61$ ), nous travaillerons principalement avec des énoncés en je. Nous nous intéresserons enfin au rôle joué par la circulation de l'information dans le fonctionnement des schèmes d'intersubjectivation. Cette étude permettra de voir comment des structures syntaxiques peuvent intégrer des contraintes extralinguistiques de nature diverse et nous conduira à proposer une description des constructions allocentrées en termes de modalité.

\section{Remarques liminaires}

\subsection{Problématisations des énoncés allocentrés}

Les énoncés allocentrés ont fait l'objet de peu d'études, du moins à notre connaissance. On peut citer ici le travail initiateur de Martin (2003) et celui de Koselak (2005) qui lui fait suite. Martin (2003) adopte une perspective vériconditionnelle et interroge les conditions de sincérité des énoncés tels $J$ 'ai honte pour toi : le locuteur éprouve-t-il véritablement l'émotion qu'il mentionne? Elle distingue les émotions normatives et les émotions empathiques : les premières sont soumises à une connaissance des normes définissant les émotions ou sentiments qu'il est approprié d'éprouver dans une situation $\mathrm{S}$; les secondes résultent de «l'adoption du point de vue d'autrui sur le monde» (Martin 2003 : 135). En comparant les énoncés Je suis honteuse pour Pierre et Je suis honteuse, l'auteure stipule que le locuteur du premier énoncé se distancie davantage de Pierre qu'il ne le fait dans le deuxième, à supposer que ce dernier soit formulé en réaction à une action honteuse de Pierre. Elle arrive à la conclusion selon laquelle les constructions en pourN inhibent l'empathie et renvoient donc exclusivement à des émotions normatives.

Si la notion de «norme» nous semble parfaitement opératoire pour l'analyse des constructions allocentrées (voir 4 ci-dessous), l'exploitation qu'en fait Martin (2003) prête le flanc à des critiques. D'une part, le choix de la honte pour illustrer ce type d'énoncés biaise l'analyse, cette émotion n'ayant pas le même statut que les autres. La honte est généralement considérée comme une émotion « sociale »" Par exemple, Koselak (2003 : 61) propose une description sémantique de la honte sous forme de structure quadri-actancielle, les deux derniers actants impliquant un lien social :

$\mathrm{X}$ avoir honte de $\mathrm{Y}$ [devant $\mathrm{Z}$ ], [par rapport à D]

$$
\begin{gathered}
\mathrm{X}=\text { le siège de la honte } \\
\mathrm{Y}=\text { la cause de la honte } \\
\mathrm{Z}=\mathrm{l}^{\text {' } ~} \text { " Autre » intériorisé, considéré comme le juge de } \mathrm{Y} \\
\mathrm{D}=\text { le système de valeurs ou de normes par rapport auquel est jugé } \mathrm{Y}
\end{gathered}
$$

Or, ce schéma actantiel ne peut pas être généralisé à l'ensemble des émotions exprimées dans des constructions allocentrées. Comme nous pourrons le voir plus bas, l'expression allocentrée de la honte s'oppose dans son contenu sémantique à l'expression allocentrée de la peur, de la joie ou de la tristesse.

D'autre part, Martin (2003) s'appuie sur une définition de l'empathie - la simulation d'autrui - qui nous semble trop étroite. Il s'agit en effet d'une définition purement praxéologique, mettant l'accent sur l'agir et les réactions du sujet s'imaginant à la place d'autrui. La définition de l'intersubjectivation que nous proposons plus haut met l'accent sur le positionnement du sujet vis-à-vis d'autrui et n'anticipe pas sur l'agir, ce qui laisse plus de place à l'analyse des formes linguistiques et n'oblige pas à rechercher l'adéquation entre réalité du langage et réalité du monde (ou du vécu de l'individu). On s'attachera ainsi à décrire les mécanismes de l'empathie linguistique, en faisant précéder le niveau interprétatif d'un niveau explicatif. 


\subsection{La notion d'empathie en linguistique}

Lorsqu'elles n'en font pas un postulat (souvent implicite) du fonctionnement du langage, comme l'énonciation (cf. la notion de co-construction, Culioli 1990) et les théories des actes du langage, de l'interaction ou de la conversation (cf. l'intersubjectivité, Vion 1992), les études linguistiques cherchent à identifier plus particulièrement les traces syntaxiques de l'empathie, qu'elles définissent comme une convergence de rôles ou de points de vue. Nous discuterons brièvement le travail de Kuno (1987), en tant que celui-ci synthétise les débuts d'une recherche linguistique sur l'empathie et ses marques langagières, et celui de Forest (1999), qui développe la problématique en France.

Kuno (1987) s'intéresse notamment aux situations d'empathisation avec l'objet ou thème du discours. Cette conception de l'empathie est proche de la notion de point de vue, l'auteur utilisant d'ailleurs la méthaphore de l'angle de vue de la caméra pour illustrer l'identification du locuteur avec l'un des participants qu'il met en scène. L'incongruïté des énoncés the alcoholic's wife $e_{i}$ killed her $_{i}$ husband ou Mary's husband talked to his wife about this problem s'explique par la saillance de alcoholic et de Mary en tant qu'éléments servant à identifier la femme et le mari respectivement. Le locuteur empathise donc avec les référents saillants. Mais l'empathie est centrée sur le sujet-locuteur, comme le souligne la règle de l'empathie des actes de langage: l'empathisation avec quelqu'un d'autre ne peut a priori être supérieure à l'empathisation avec soi-même (?? John m'a rencontré hier à la réception) (Kuno 1987 : 212). L'anaphore, les réfléchis, les verbes come up to vs go up to constituent quelques-unes des marques linguistiques d'empathisation analysées par Kuno (1987).

Forest (1999: 22) voit l'empathie come un domaine énonciatif, s'organisant autour de la question de l'énonceur omniscient ou non. Le moyen (voix moyenne), la version en géorgien et les verbes aller/venir sont considérés par l'auteur comme des traces privilégiées de l'empathie.

La version en géorgien est représentée par une voyelle marquant l'orientation du procès : «En tant que morphème empathique, la marque de version sert à mettre en perspective le procès (par ailleurs actualisé) en l'orientant vers le 'concernement' d'une entité.» (Forest 1999: 153). On distingue la version subjective, objective, suppressive ou neutre. La version subjective, marquée par l'insertion de la voyelle $i$ devant des verbes transitifs, montre soit que le sujet agit sur lui-même ou dans son propre intérêt, soit qu'un patient ou bénéficiaire de la $1^{\text {ère }}$ ou $2^{\text {ème }}$ personne est concerné par l'action (Forest 1999: 135-136). La version subjective, marquée par la voyelle $u$, signale que le concerné n'est ni le locuteur, ni l'allocutaire.

La voix moyenne (représentée facilement par des réfléchis en français : se dégeler) et des formes proches tel que le datif étique (je me le ferais bien) sont caractérisées par la fusion du point de départ et du point d'arrivé du procès, ce qui produit une solidarité entre agent et patient ou bénéficiaire. Nous reviendrons plus bas (2.2.) sur le fonds empathique de la voix moyenne, autour des verbes pronominaux utilisés dans les constructions allocentrées.

Le survol de ces travaux fait ressortir quelques axes centraux à la problématique de l'empathie. D'une part, on voit se crystalliser la notion de « solidarité », qui est transposable aux constructions allocentrées. D'autre part, on notera le pontentiel explicatif des rôles sémantiques, mobilisés pour la description des réfléchis, de la voix moyenne et, dans une moindre mesure, de la version. Enfin, c'est le statut cardinal de l'énonciateur qui ressort. En se positionnant, l'énonciateur marque les discours de la relation entre Soi et Autrui qui définit l'empathie. Le sujet parlant participe de ce fait au partage social des émotions, défini par Rimé (2005) comme un processus disposant d'un schéma énonciatif dont les rôles prototypiques sont assumés par le sujet expérimentateur de l'émotion et par son allocutaire singulier ou pluriel. Reste à voir la place que les constructions allocentrées occupent dans ce dispositif. 


\section{Description syntactico-sémantique}

\subsection{Double sélection des constructions allocentrées}

Les constructions allocentrées possèdent une série de caractéristiques les rendant plus ou moins homogènes et justifiant leur regroupement en une classe à part. Ainsi, s'il existe de nombreuses structures en pourN, elles n'ont pas la même valeur ni les mêmes conditions d'interprétation. Dans les exemples (construits) ci-dessous, nous comparons les trois formats grammaticaux des constructions allocentrées à des structures syntaxiques diverses respectant le même format.

(4) Il travaille pour son voisin

Versus

(5) Il craint/s'inquiète [fortement] pour X [+humain]

Dans les deux énoncés (4) et (5), un sujet humain se combine avec un verbe suivi de pourN[+humain]. Cependant, la valeur sémantique n'est pas du tout la même: dans le premier, il s'agit d'une action effectuée «pour le compte de/au bénéfice de », dans le second, d'une émotion vécue « en rapport avec/à la place de $»$.

(6) Elle est (trop) jeune pour lui

Versus

(7) Elle est [très ; toujours ?] contente / triste pour X

Les contraintes sémantico-syntaxiques sont respectées dans les énoncés (6) et (7) également. Mais on voit que, d'une part, les intensifs susceptibles d'intégrer ces constructions ne sont pas nécessairement les mêmes et, d'autre part, le groupe prépositionnel enregistre, là encore, des valeurs sémantiques différentes - « par rapport à lui/compte tenu de son âge (valeur contrastive) » en (6) et « en rapport avec/en ce qui concerne (valeur associative) » en (7).

Ces deux premiers couples d'énoncés montrent que les termes d'émotion disposent, dans le cadre des formats étudiés, d'un statut à part, ce qui permet de distinguer les constructions allocentrées des autres constructions syntaxiquement proches.

Enfin, les énoncés (8) et (9) mettent au jour la spécificité des émotions allocentrées par rapport à d'autres termes de sentiments ou d'émotion :

(8) J'ai de l'amitié / de l'amour / de la haine / du mépris etc. pour X

Versus

(9) J'ai peur, honte pour $\mathrm{X}$

Ainsi, les termes réciproques (être ami avec qqn, aimer qqn, se haïr) sont quantifiés par un partitif (avoir de l'amour, du mépris), pourN[+humain] renvoyant en (8) à l'objet bénéficiaire du sentiment ou de l'émotion ; (9) a une interprétation comparable à celles de (5) et de (7).

Au niveau référentiel, on notera que les constructions allocentrées n'intègrent que quatre émotions, dont les trois premières sont des émotions de base $^{6}$ (Ekman 1980) - peur, joie, tristesse, honte - dans nombre de leurs expressions lexico-syntaxiques (ainsi, pour la peur, on aura s'inquiéter, s'affoler, craindre, avoir peur, etc.). On peut mentionner cependant trois autres expressions (cf. Martin 2003) : je suis fier pour toi (plutôt rare, autour de 3000 occurrences sur Google ${ }^{7}$; proche de content), je suis embarrassé pour toi (une seule occurrence sur Google), je suis déçu pour toi (qui relève du même domaine émotionnel que la tristesse), très proches des constructions allocentrées mais qui ne sont pas ambigües ${ }^{8}$, dans le sens où l'expression semble remplir d'emblée une « condition de sincérité » quant à l'expérience émotionnelle du sujet parlant. 


\subsection{Formats des expressions émotionnelles allocentrées}

Reste à étudier les formats des expressions émotionnelles et leur place dans les constructions allocentrées : avoir+terme d'émotion, être+terme d'émotion, verbe, verbe pronominal.

Timérique (peur)
AVOIR+N (avoir peur)
V Pronominal (s' inquiéter)
ETRE+Adj (être inquiet)
V (?craindre, *redouter)

Honte
AVOIR+N (avoir
honte)

\begin{tabular}{|l|l|}
\hline Joie & Tristesse \\
\hline $\begin{array}{l}\text { ETRE+Adj (être } \\
\text { content) }\end{array}$ & ETRE+Adj (être triste) \\
$\begin{array}{l}\text { V Pronominal (se } \\
\text { réjouir) }\end{array}$ & \\
\hline
\end{tabular}

Tableau 1. Formats syntaxiques des émotions et constructions allocentrées.

Les constructions allocentrées sélectionnent une série d'expressions lexico-syntaxiques et acceptent tous les formats excepté le Verbe non pronominal : redouter, effrayer, angoisser sont en principe exclus de ces constructions, à cause de leur statut non pronominal et de leur valence, ou encore des rôles sémantiques qu'ils intègrent (cf. la section 3 pour une analyse détaillée). Craindre, bien qu'intégrant des structures en pourNhumain, demande la présence de $N$ non humains qui modifient l'interprétation du Nhumain :

(10) non, je crains pour toi l'humidité du ruisseau, tu as toussé aujourd'hui. (MAURIAC François, Asmodée, 1938, p. 64, ACTE II scène 3)

Les particularités sémantiques des trois formats intégrés par les constructions allocentrées - avoir +terme d'émotion, être+terme d'émotion, verbe pronominal - pourraient-elles apporter un éclairage quant à la nature de ces dernières ? Benveniste explique bien la différence entre les deux verbes auxiliaires :

Etre est l'état de l'étant, de celui qui est quelque chose; avoir est l'état de l'ayant, de celui à qui quelque chose est. [...] Entre les deux termes qu'il joint, être établit un rapport intrinsèque d'identité : c'est l'état consubstantiel. Au contraire, les deux termes joints par avoir demeurent distincts ; entre ceux-ci le rapport est extrinsèque et se définit comme pertinentiel ; c'est le rapport du possédé au possesseur. (Benveniste $1966: 198)$

Le verbe d'émotion pronominal est exempt quant à lui de toute réflexivité proprement dite qui ferait du pronom réfléchi le COD du verbe et le patient de l'action. Mais il semble avoir une dimension intentionnelle que ne partagent pas les verbes non pronominaux.

On peut décrire les trois formats dans les termes suivants :

- $\quad \underline{A V O I R+N}$ : localisation-possession (le sujet comme lieu, siège de l'émotion nommée ; propriété essentielle selon Leeman 1995);

- ETRE+Adj. : caractérisation (le sujet comme affecté $\rightarrow$ siège de l'émotion ; état résultatif selon Leeman 1995);

- Verbe pronominal : intentionnalité relative, le sujet comme siège de l'émotion.

L'opposition entre les formats en « être » et les formats en « avoir » n'est pas pertinente en soi car chacun d'eux est associé à une émotion particulière : joie//tristesse et peur//honte respectivement. Leur coloration sémantique pourrait en revanche être exploitée pour expliquer d'éventuelles différences entre l'expression allocentrée de la joie et de la peur, par exemple.

A la différence des formats en «être » et en «avoir», les verbes pronominaux ne représentent pas un mode d'expression spécifique d'une émotion mais constituent des alternatives à ces formats pour exprimer la peur, la joie ou la tristesse. De ce fait, leur analyse contribuerait à apporter des explications quant au fonctionnement des constructions allocentrées. Ces verbes n'impliquant pas une réflexivité classique à l'instar de se dénoncer, l'emploi du pronom réfléchi influe sur le statut du sujet grammatical, susceptible d'articuler siège et bénéficiare de l'action, comme c'est le cas dans la voix moyenne (voir Forest 1999). Une telle convergence relève des stratégies d'empathisation. C'est chez Herslund (2007) que l'on trouve, nous semble-t-il, une explication appropriée du fonctionnement de ces pronominaux. Selon cet auteur, on a affaire à un antipassif réfléchi complexe, qui a pour effet la mise en relief ou la «promotion d'un agent ou d'un expérient» (Herslund 2007 : 175). Dans le cas des verbes d'émotion 
comme s'inquiéter ou se réjouir, le réfléchi signalerait donc une implication intentionnelle (on dirait presque, sans jeu de mots, réfléchie) du sujet-siège dans le procès verbal, une sorte d'engagement subjectif .

Le rôle sémantique de siège assumé par le sujet semble essentiel - en plus des contraintes mentionnées plus haut - à la configuration des constructions allocentrées. Nous proposons une description détaillée des rôles propres à ces constructions dans la section 3 .

\subsection{Interprétation de pourN}

La construction en pour des quatre structures mentionnées soulève la question du type de relation que la préposition sémiotise. Pour Martin (2003: 131), le complément en pour exprime soit le bénéficiaire (j'ai voté pour Zébulon), soit l'agent auquel le sujet se substitue (j'ai voté à la place de Zébulon). Cadiot (2007) propose quant à lui quatre séries interprétatives de la phrase-type Paul est triste (,) pour Marie que nous reproduisons dans le tableau ci-dessous. Toutes impliquent une altérité, qu'elle soit énonciative, thématique ou agentive: la construction pourN se place indubitablement sur le terrain de l'intersubjectivité.

\begin{tabular}{|l|l|l|l|}
\hline (a) ext. & Incidence à l'énonciation & \pm selon, d'après & $=$ point de vue \\
\hline (b) ext. & Incidence à l'énoncé & \pm en ce qui concerne & $=$ thème discursif \\
\hline (c) int. & Incidence au prédicat & \pm par égard pour & $=$ participatif \\
\hline (d) int. & Alternance en position-argument & \pm à la place & $=$ équatif \\
\hline
\end{tabular}

Tableau 2. Lectures de pourN. (Cadiot 2007 : 82)

Ces lectures ne sont pas nécessairement étanches, elles «sont éventuellement appelées à fusionner (par coalescence) dans certaines configurations comme le clivage : C'est pour Marie que Paul est triste » (Cadiot 2007 : 82). Dans ce cas de figure, Cadiot (ibidem) reconnaît la fusion des lectures $b+c$ ou $b+d$. Or il nous semble que rien ne s'oppose à un rapprochement plus global entre b, $c$ et d, et plus particulièrement entre $c$ et $d$, dans une optique d'intersubjectivation empathique. Nous tâcherons de dégager, dans ce qui suit, les principes sous-tendant de telles interprétations convergentes.

\section{Analyse des rôles sémantiques : siège, stimulus, situation et le tiers- actant}

Les constructions allocentrées ont une configuration actantielle qui demande à être située dans le contexte et à être spécifiée en vue d'une interprétation sémantique détaillée. Premièrement, il s'agit d'éclairer le statut de $\operatorname{pourN}$, qui ne s'identifie pas au sujet-siège de l'émotion mais qui constitue une sorte de tiersactant se greffant sur le siège et le stimulus. Deuxièmement, il convient de préciser le rôle du stimulus et de la situation dans l'expression émotionnelle. Au final, l'objectif est de définir les places de chacun des éléments configurant sémantiquement l'affect : siège, stimulus, situation (les trois $\mathrm{S}$ ), et leur rapport au tiers-actant.

\subsection{Le tiers-actant dans les constructions allocentrées et le rapport siège- stimulus}

Si l'interprétation de pourN (ci-dessus) permet de spécifier l'attitude du sujet-siège envers le tiers-actant et de préciser les types de relations que ces derniers entretiennent au sein de l'énoncé, le statut de ce tiersactant reste à définir.

Il convient d'abord de vérifier si ce dernier ne se confond pas avec le stimulus de l'émotion; pour ce faire, le tiers-actant est placé en position sujet ${ }^{10}$ dans des structures causatives dont on évalue la 
possibilité d'une équivalence avec les constructions allocentrées. Corollairement, ces structures font ressortir la prise en charge émotionnelle par le sujet parlant, grâce à l'adjectif attribut ${ }^{11}$.

Timérique
Tu me fais peur
Tu m'inquiètes
Tu m'effraies
\#
J'ai peur pour toi

\begin{tabular}{l|l}
\hline Honte & Joie \\
\hline Tu me fais honte & Tu me rends heureux \\
$\approx$ & $\neq$ \\
J'ai honte pour toi & Je suis heureux/content \\
& pour toi
\end{tabular}

Tableau 3. Structures causatives : tiers-actant et stimulus.

\section{Tristesse}

Tu me rends triste $? ? ?=$

Je suis triste pour toi

Le tiers-actant ne semble pas se confondre avec le stimulus, les structures causatives n'étant pas équivalentes aux constructions allocentrées (même si des hésitations interprétatives persistent, nous y reviendrons plus bas).

Pour ce qui est de la peur, des alternances lexicales sont prévues pour rendre compte du type de rapport établi entre le siège et le stimulus. Par exemple, Kay (2003) analyse dans le détail l'opposition entre 11, où l'affecté fonctionne comme sujet actif, et 12, où le stimulus fonctionne comme sujet actif ${ }^{12}$ :

$$
\begin{aligned}
& \text { (11) Robert }{ }^{\text {Siège (affecté) }} \text { craint les ours }^{\text {Contenu }} \text {. } \\
& \text { (12) Les ours }{ }^{\text {Stimulus }} \text { effrayent Robert }{ }^{\text {Siège (affecté) }}
\end{aligned}
$$

La répartition des rôles sémantiques entre le sujet et le COD détermine entre autres la possibilité d'utiliser tel ou tel lexème dans les constructions allocentrées ; ainsi, lorsque le stimulus fonctionne comme sujet, l'expression allocentrée est impossible, et pour cause : il faut que le siège de l'émotion conserve son potentiel intentionnel pour qu'une place soit faite au tiers-actant.

L'expression de la joie signale des écarts d'intensité possibles voire des changements émotionnels (contentement et bonheur relèvent-ils de la même catégorie émotionnelle ?), comme on peut le voir dans le tableau.

Le cas des expressions de la honte et de la tristesse semble plus complexe. En effet, la relation entre le tiers-actant et le stimulus y est ambiguë, l'empathie pouvant produire un amalgame entre le tiers-actant et le stimulus notamment dans le cas de la tristesse. Dans le cas de la honte, c'est la construction $j$ 'ai honte de toi qui semble être le pendant de la structure causative.

La causalité est directement liée à une dimension évaluative : il s'agit d'évaluer l'impact potentiel d'une entité sur l'état émotionnel du sujet. Une certaine subjectivité reste présente ici, car l'évaluation rend compte de l'attitude subjective (de l'adhésion) par rapport à un système de normes ${ }^{13}$. L'étude des énoncés présentatifs et intensifs nous permettra de préciser le rôle de l'évaluation dans l'expression allocentrée des émotions.

\subsection{De la situation à l'émotion : les énoncés évaluatifs}

Les constructions allocentrées sont souvent reliées en contexte, de manière implicite ou explicite, à des évaluations représentées par des énoncés présentatifs ( $C$ 'est triste) ou expressifs intensifs (Quelle honte !) qui qualifient la situation à l'origine de/provoquant l'émotion. Ces rapports contextuels peuvent être relativement éloignés en discours et s'organiser au sein d'une activité argumentative, comme cela a pu être montré par Cislaru (2008). Par exemple, les rapports rédigés par les travailleurs sociaux pour évaluer la situation d'enfants en risque de danger s'organisent, au niveau argumentatif, de la manière suivante : L'enfant a peur (facultatif) $\rightarrow$ Nous nous inquiétons pour l'enfant (très régulier; = à la place de l'enfant ?) $\rightarrow$ La situation est inquiétante, l'administration doit intervenir (au cas par cas). On constate donc que l'expression allocentrée est directement liée à une interprétation-évaluation de la situation ${ }^{14}$ qui valide l'expression émotionnelle. Dans le cas des constructions allocentrées, l'évaluation de la situation est directement liée à la modalité épistémique (évidentialité) dont il sera question plus bas. 
Le tableau ci-dessous recense les énoncés présentatifs et expressifs concernant chacune des émotions représentées par des constructions allocentrées.

\begin{tabular}{|l|l|l|l|}
\hline Timérique & Honte & Joie & Tristesse \\
\hline $\begin{array}{l}\text { C'est affreux! } \\
\text { C'est inquiétant }\end{array}$ & C'est honteux & C'est génial/super ! (?) & C'est triste \\
\hline C'est regrettable
\end{tabular}

Tableau 4. Présentatifs et expressifs.

Cette présentation permet de mettre au jour les éléments du contexte sur lesquels porte l'évaluation. Ainsi, dans le cas de la peur, de la tristesse et de la joie, c'est plus particulièrement le stimulus de l'émotion (événement ou situation) qui est soumis à l'évaluation. Dans le cas de la honte, c'est plutôt le comportement de la personne qui est visé (le tiers-actant donc). Signalons toutefois qu'il n'est pas impossible de faire porter l'évaluation concernant la peur notamment, mais aussi la joie et la tristesse, sur le comportement: imaginons un je m'inquiète pour lui glissé par une mère au sujet de son enfant trop timide la veille de sa première rentrée à l'école.

On repère des alternances lexicales entre les présentatifs et les évaluatifs, qui permettent d'affiner l'interprétation sémantique tout en intégrant un nouveau trait sémantique, l'aspectualité. Les émotions timériques (champ de la peur) sont celles qui distinguent le plus nettement les ingrédients de cette expérience émotionnelle : siège et stimulus sont clairement départagés (comme stimulus et tiers-actant d'ailleurs, voir ci-dessus), de même que l'aspectualité (duratif $><$ ponctuel) est prise en compte, ce qui permet de distinguer les états et traits de caractère (duratif) des expériences situationnelles nécessitant généralement de spécifier un stimulus (ponctuel).

$$
\begin{aligned}
& \text { (13) C'est affreux }{ }^{\text {cause-situation }} \\
& \text { (14) Je suis peureux }{ }^{\text {siège-duratif }} \\
& \text { (15) Je suis inquiet }{ }^{\text {sìge-ponctuel/situationnel }}
\end{aligned}
$$

Le domaine de la joie accuse quelques trous lexicaux, comme le montrent les structures présentatives non apparentées. Mais l'opposition duratif-ponctuel y est également marquée, avec cependant une hésitation quant à l'identité émotionnelle, comme on a déjà pu le remarquer plus haut :

(16) Je suis joyeux ${ }^{15} /$ enjoué $e^{\text {siège-duratif }}$

(17) Je suis content $t^{\text {siège-ponctuel/situationnel }}$

L'expression de la honte ne permet pas de faire ce genre de distinctions : on dispose en français d'un seul adjectif qui active les mêmes traits, quel que soit l'objet qualifié :

(18) C'est honteux ${ }^{\text {cause-comportement }}$

(19) Je suis honteux $x^{\text {sì̀ge-agent comportemental }}$

Il en est de même pour l'expression de la tristesse qui ne marque pas les différences sémantiques mentionnées plus haut (je suis triste/c'est triste).

L'évaluation permet, d'une part, de justifier l'expression émotionnelle, d'autre part, de distinguer les trois $\mathrm{S}$ (siège, stimulus, situation) tout en les départageant du tiers-actant dans trois cas sur les quatre étudiés.

\subsection{Statut du tiers-actant}

Syntaxiquement et sémantiquement parlant, le tiers-actant ne se confond pas avec le siège de l'émotion. Il apparaît plutôt comme une sorte de bénéficiaire de fait de l'émotion exprimée par le sujet parlant. Cependant, dans le schéma de Cadiot ci-dessus, le niveau d) d'interprétation prévoit un équatif, une 
possibilité de substitution référentielle donc. Dans cette lecture, le siège de l'émotion initial serait le tiersactant, auquel l'énonciateur ne ferait que se substituer. L'étymologie permet de faire quelques parallèles en faveur d'une telle interprétation : ainsi, l'un des équivalents pragmatiques de Je suis triste pour toi mes condoléances - est marqué étymologiquement par la réciprocité due aux préfixes con-/com- en latin (condolesco = « souffrir ensemble », compatir, cf. Zaliznjak et Shmelev $2007: 210$ ).

La substitution référentielle du siège au tiers-actant implique une sorte de dédoublement du siège de l'émotion qui correspond de fait à l'empathie. Reste à vérifier si l'on a véritablement affaire à une expérience commune et, si tel est le cas, à déterminer les voies par lesquelles ce partage est réalisable.

Les quatre émotions intégrant des constructions allocentrées n'ont pas le même degré de prise en charge, d'après les données sémantiques et discursives. Ainsi, la tristesse et la joie présupposent une expérience émotionnelle identique pour le tiers-actant et pour le sujet-siège : il est d'ailleurs pragmatiquement insolite - moquerie, ironie, etc. - de dire je suis triste pour lui de quelqu'un qui vient de se marier ou qui vient d'avoir une promotion. La peur est ambivalente: le tiers-actant n'est pas censé ressentir nécessairement la même émotion que le sujet-siège - on peut avoir peur pour un bébé qui n'a pas a priori conscience d'un danger précis, mais cela reste une potentialité. Par exemple, dans certains corpus tels les rapports sociaux dans le cadre du signalement d'enfant en danger, la fusion expérientielle est de mise, ou du moins suggérée comme telle par les enchaînements textuels (voir Cislaru 2008 : 128-129) :

(20)L. et T. ont peur de leur père et ne souhaitent plus le voir.

(21) Nous ne pouvons qu'être inquiets quant à l'évolution de L. et T.

(22)Tout ce que j'espère, c'est que celle-ci soit plus amène [sic] d'accueillir $\mathrm{J}$. dans de bonne condition psychologique pour elle et surtout pour mon fils car je crains que la situation ne se dégrade et cela est légitime de pouvoir y penser, mais il subit les agissements irresponsables de sa maman.

Ces trois énoncés sont tirés du même rapport social et sont présentés dans l'ordre de leur apparition dans le texte.

Dans d'autres corpus comme la presse, les forums ou les numéros verts Internet, l'information concernant l'expérience émotionnelle du tiers-actant est absente ou négative :

(23) Inquiète pour une amie, Fille 12 ans

Bonjour tel-jeune si je vous écrie c'est que j'ai un grave problème. Une de mes amies se mutille et elle ma dit sur msn qu'elle avait le cancer. Je la crois mais j'ai peur que ça sois vraie. Mais pour la mutillation elle ne ment pas car j'ai vue ses cicatrices. Aussi on ne se connais pas depuis longtemps et notre amitiée n'est pas encore très dévloppée alors j'ai peur de lui en parler. Que faire?? Une amie Paniquée (TelJeunes Canada)

La honte est un cas à part. Son expérience par le tiers actant n'est pas requise, elle est, au contraire, supposée absente chez ce dernier (et c'est précisément cette absence qui « produit» la honte chez le sujet locuteur) :

\section{(24) J'ai honte pour lui}

la chaise electrice serait trop douce pour lui il faut qu'il se souvienne de ce qu'il a fait a son chien

(Posté par gigi17, 15 janvier 2009 http://savetheplanet.canalblog.com/archives/2007/04/10/4583092.html)

On constate que, si expérience commune il y a entre le siège et le tiers-actant, elle ne concerne pas l'ensemble des quatre émotions apparaissant dans des constructions allocentrées. Ce constat appelle plusieurs éclaircissements qui seront apportés plus bas. Premièrement, en ce qui concerne la valeur des constructions allocentrées et leur regroupement au sein d'une même catégorie alors même que le partage expérienciel fait défaut dans certains cas. Deuxièmement, en ce qui concerne les modalités de partage expérienciel, si partage il y a. Enfin, il serait intéressant de mettre en relation les écarts observés plus haut entre les quatre émotions en termes de rapports siège-stimulus-situation et la valeur des constructions allocentrées. 


\section{Intersubjectivation empathique ou pragmatique?}

\subsection{Evidentialité ${ }^{16}$, inférence et partage expérienciel}

Nous nous pencherons pour commencer sur les modalités de partage expérienciel. Linguistiquement parlant, les émotions sont rattachées au sujet (je), comme le montre l'interprétation absolument univoque d'un syntagme nominal Dét. Poss $+\psi_{N}$ (nom psycologique, Anscombre 1995 : 43) : cf. par exemple, ma peur (siège, lieu psychologique) et mon dictionnaire (possesseur vs auteur). En même temps, on dispose souvent de données paralinguistiques permettant d'interpréter, ne serait-ce que partiellement, les émotions des autres. Ces données sont largement exploitées dans les discours qui emploient des constructions allocentrées :

(25) A ce moment-là, L. se raidit, son visage s'assombrit. Elle attend la suite de notre raisonnement, en donnant le sentiment d'avoir peur de la proposition. (enquête sociale)

Plus rarement, on fait usage de formes diverses de discours rapporté :

- Discours direct :

(26) Dans la classe des CM2, une fillette brune confie : « [...] On disait les États-Unis intouchables, maintenant j’ai peur. On peut toucher n'importe quel pays. » (Le Monde, 15/09/01)

- Discours indirect, avec éventuellement un terme d'émotion en lieu et place du verbe de parole :

(27)Les spécialistes craignent que ce virus ne passe chez d'autres espèces (Le Figaro, 11/06/03)

(28) Malgré les appels au calme, la communauté s’inquiète des amalgames (Libération, 17/09/01)

- Discours narrativisé ou indirect libre, comme le montre le contexte large et le sous-genre « entretien » de l'exemple (20) ci-dessus.

On évoque donc des informations et des sources d'informations susceptibles d'expliciter et de confirmer l'expérience émotionnelle du sujet. L'expression émotionnelle dans les constructions allocentrées est alors liée à un raisonnement du type « $\mathrm{X}$ parce que je le vois/entends » ou « $\mathrm{X}$ parce que $\mathrm{Y}$ dit $\mathrm{X}$ »; le premier relève de l'évidentialité perceptive ou inférentielle, le deuxième de l'évidentialité d'emprunt, s'assimilant ici à l'argument d'autorité par témoignage. Cette accessibilité expériencielle constitue la première condition - suffisante, mais non nécessaire - d'une empathisation ultérieure dans le cadre des constructions allocentrées.

Dans d'autres contextes, cependant, ces données directes peuvent faire défaut. Mais cela ne bloque pas pour autant la possibilité d'utilisation des constructions allocentrées, d'autres éléments étant intégrés au raisonnement : les données situationnelles et causales ${ }^{17}$. Sont alors évoqués le stimulus de l'émotion et des informations sur la situation :

(29) Lens : Monterrubio triste pour son ancien $\operatorname{club}^{18}$

Le néo-Merlu ne cache pas une certaine émotion, au vu de la passe difficile traversée par les Artésiens, actuels $19 \mathrm{es} \mathrm{du} \mathrm{classement.} \mathrm{(maxifoot.fr,} \mathrm{30/10/09)}$

(30) OM-Deschamps : " Content pour Gaby »

Unique buteur de la victoire marseillaise à Zurich (1-0) en ligue des Champions, Gabriel Heinze, arrière gauche mercredi soir, a réjoui son entraîneur Didier Deschamps. " En l'alignant à gauche, je ne pensais pas qu'il allait marquer, et encore moins du pied droit », a confié le coach marseillais à Canal+. «Je suis content pour lui, je suis content pour Lucho, content pour l'équipe. Je fais des choix, j'ai un bon groupe [...]» (Ouest France, 21/10/09)

Les constructions allocentrées prennent alors appui sur un raisonnement inférentiel du type «X parce que je sais que $\mathrm{Y}$, et $\mathrm{Y}$ implique $\mathrm{X} »$. On a affaire à une référence déductive, une situation $\mathrm{X}$ produisant (nécessairement?) l'émotion Y. L'évaluation s'appuie sur une conception assez générale de ce qui est bien (susceptible de ce fait de produire des émotions positives) et de ce qui est mal (susceptible de produire des émotions négatives) ${ }^{19}$. 
Il n'est pas rare que la construction allocentrée serve de titre d'article, de blog (cf. 30) ou de message (cf. 24), en cela constituant une sorte de synthèse du contenu textuel. Il convient par ailleurs de souligner que, en tant que titres d'article, ces constructions constituent le résultat du travail interprétatif du rédacteur.

\subsection{Modalité épistémique et modalité déontique}

On vient d'observer des opérations de création d'information qui semblent mettre en relation expérience émotionnelle et savoirs collectivement partagés. Subjectivité versus objectivité ? Nuyts (2001) propose un modèle d'(inter)subjectivité comme une alternative à l'opposition subjectivité/objectivité de Lyons (1977). Il oppose les expressions de la subjectivité (in my view/opinion/mind, if you ask me, to me...) aux expressions marquant l'intersubjectivité ${ }^{20}$ (it is known that) (Idem, 387). En effet, si l'énonciateur semble inférer l'expérience émotionnelle à partir de données situationnelles, ces données en elles mêmes reçoivent une valeur intersubjective relevant de l'ontologie à la troisième personne (on), de l'emprunt, plutôt que de l'ontologie à la première personne ${ }^{21}(j e)$. L'information émotionnelle est accessible par intersubjectivation : il existe des sortes de codes émotionnels interprétatifs pour des séries de situations. On peut aborder par ce biais les hypothèses du locuteur concernant les connaissances et les savoirs de l'interlocuteur ou du tiers-actant, si celui-ci ne s'identifie pas avec l'interlocuteur. Ces connaissances et savoirs relèvent du domaine de l'intersubjectivité et renvoient à des normes au sens de Kronning (1996) :

Ces régularités nominiques [= modèles cognitifs idéalisés, de type propositionnel] peuvent être de nature extrêmement diverse : normes prescriptives, telles que les lois juridiques et religieuses, normes constitutives, telles que les règles d'un jeu ou de la grammaire, normes téléologiques, telles que les mœurs, les coutumes et les habitudes, lois morales, lois logiques et mathématiques, lois de la nature, scénarios, lieux communs, topoï, etc. (Kronning $1996: 35$ )

L'inférence déductive est ainsi basée sur une série de stéréotypes sociaux (on est content quand on trouve du travail, par exemple) concernant les sources et les cadres des émotions : il doit avoir peur car la situation est inquiétante, dangereuse, etc. $\rightarrow j$ 'ai peur pour lui; il doit être triste car les événements sont négatifs $\rightarrow$ je suis triste pour lui :

(31) - Je suis désolée, dis-je.

Je le suis sincèrement pour lui parce que si Pierre Arthens ne souffre plus, il va falloir à Chabrot apprendre à vivre en étant comme mort. (BARBERY Muriel, L'élégance du hérisson, 2006, Gallimard, p. 125)

Dans cette démarche interprétative - qui relève d'une conception évaluative des émotions (Gibbard 1996 : 165 et $s q$.) - semble s'imposer la proximité des opérations inférentielles analysées plus haut avec le devoir $_{E}$ (devoir épistémique) tel qu'il est analysé par exemple dans les travaux sur l'évidentialité de Dendale (1994), même si le verbe épistémique reste implicite dans les constructions allocentrées

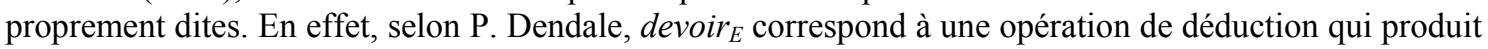
ici de l'information permettant de justifier le déclenchement de l'empathie. La peur, la tristesse et la joie allocentrées prennent ainsi appui sur la modalité épistémique implicite.

La honte, en revanche, ne suit pas le même scénario interprétatif. L'enchaînement il doit avoir honte $\rightarrow$ j'ai honte pour lui n'est pas valide, comme le montrent les analyses proposées dans la section 3. C'est le conditionnel du verbe devoir qui s'impose dans le cas de la honte, tout en transformant la valeur

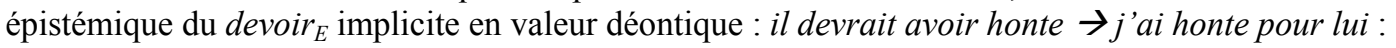

(32) Pour être tout à fait honnête, j'avais "zappé". Je devrais avoir honte ${ }^{22}$ ... (mail personnel)

La modalité déontique prend appui sur des normes sociales de comportement bien définies (voir Kronning plus haut), cet avis étant partagé par plusieurs auteurs travaillant sur l'expression des émotions qui nous préoccupent ici. Dans son étude de l'expression de la honte ou de l'embarras «pour le compte d'autrui », Martin (2003 : 132) propose de distinguer les émotions normatives des émotions privées, pour signifier que le sujet se conforme dans ce cas à la norme émotionnelle instanciée ${ }^{23}$. Koselak va jusqu'à intégrer la norme sociale dans la description sémantique de la honte en proposant une structure sémantique quadri-actancielle, dont le quatrième actant est « le système de valeurs ou de normes par 
rapport auquel est jugé Y» (Koselak 2003: 61), ou la doxa (Koselak 2005). Les appréciatifs accompagnant les constructions allocentrées sont des marqueurs de cette norme sociale :

(33) En présentant Fadela Amara, secrétaire d'Etat chargée de la Politique de la Ville qui lui est désormais rattachée, Brice Hortefeux a lâché ces quelques mots : c'est « une compatriote » avant d'ajouter « comme ce n'est pas forcément évident, je le précise. »

Europe 1. Enfin, on voit le fond abjecte de la pensée de beaucoup de français, et en particulier de ce gouvernement. J'ai honte pour lui, j'ai honte pour elle.

(Parle roncier@10:04 AM; http://le.roncier.net/)

(34)@, Manu : si si, moi j'les ai vus au loin! Alors la jeune dame m'a paru très bien équipée et parée pour l'aventure, par contre Thomas il avait un appareil à raclette sur le dos, et 3 caisses de vin de pissenlit... Je crois qu'en fait il pensait faire une bouffe entre potes. C'est portenawak. Et en plus il s'est aussi épilé le crâne et il a un bonnet péruvien. Il s'est gourré de pays. J'ai honte pour lui...

(http://jesuispasunmecmarrant.wordpress.com/2008/11/01/pourquoi-jai-enormement-de-respectpour-les-femmes/)

On prend la mesure des différences qui opposent l'expression allocentrée de la honte aux autres constructions allocentrées ; on distinguera l'intersubjectivité empathique propre au trio peur-joie-tristesse, basée sur la modalité épistémique, et l'intersubjectivité pragmatique ${ }^{24}$ propre à la honte - et, dans certains cas, à la peur - basée sur la modalité déontique. Cette dernière s'apparente à un acte illocutoire, soustendu par la modalité déontique :

\begin{abstract}
« Deontic modality, however, expresses the speaker's moral assessment of the state of affairs, but also involves imposing this upon the agent/subject of the clause (as one participant in the state of affairs expresses), i.e., it is both speaker- and subject-oriented. Epistemic modality, finally, has little or nothing to do with the internal situation of the state of affairs (e.g., it is not specifically related to the subject of the clause), but involves a speaker's evaluation of the 'ontological' status of the state of affairs, i.e., it is entirely speaker-oriented. » (Nuyts 2001: 398)
\end{abstract}

Les constructions allocentrées autour de la peur, de la joie et de la tristesse s'apparentent quant à elles à des actes perlocutoires jouant un rôle significatif dans le partage social des émotions (cf. Rimé 2005), comme le montre aussi la fréquence de ces structures dans le discours du sport, dans les écrits sociaux, dans les espaces d'échange en ligne.

La distinction qu'on vient de faire entre intersubjectivité empathique et intersubjectivité pragmatique, bien que descriptivement opportune, demande quelques ajustements. Car les codes sociaux représentent par ailleurs non seulement une ressource évaluative, mais aussi un potentiel de contrainte. La valeur épistémique des constructions allocentrées n'empêche pas le transfert vers une valeur déontique, telle l' « obligation » d'être heureux lors de son mariage, l' « obligation » d'être triste dès lors qu'un proche est décédé, etc., toujours sous l'impact/pression des stéréotypes et des normes sociales. Le fait que l'empathie s'appuie sur une inférence déductive (logiquement nécessaire, donc) pourrait expliquer le basculement possible de l'épistémique dans le déontique.

\title{
5 Conclusions
}

Nous avons proposé dans cette étude une description sémantico-énonciative détaillée des constructions allocentrées du type N1humain+expression émotionnelle+pour+N2humain (Je suis content pour toi), en tant que celles-ci rendent compte du potentiel intersubjectif du langage tout en mettant au jour les mécanismes qui facilitent sa réalisation.

L'analyse sémantico-syntaxique montre que seules les expressions émotionnelles accordant le rôle de siège au sujet grammatical et acceptant un complément non obligatoire à rôle de stimulus peuvent intégrer ce type de constructions. L'étude des rôles sémantiques des deux noms humains est apparue dès lors comme déterminante pour l'identification des contraintes sémantiques. On a pu identifier des rôles sémantiques actualisés (siège, tiers-actant) et des rôles sémantiques implicites ou inférentiels (stimulus, directement dépendant de la situation). La fusion expérientielle entre le siège et le tiers-actant apparaît 
comme aléatoire dans l'ensemble, et négative dans le cas de la honte. Pour analyser les rôles sémantiques implicites, nous avons dû faire appel à des cadres énonciatifs aptes à prendre en charge la pratique d'évaluation de la situation, cruciale dans l'expression allocentrée des émotions. L'analyse en termes d'évidentialité, prise au sens de Dendale (1994) notamment, met au jour des contraintes sociales et culturelles se manifestant sous deux formes : le savoir et le devoir.

Le schéma sémantique et les schèmes d'intersubjectivation apparaissent ainsi comme directement liés, la possibilité de fusion siège-tiers-actant se rattachant à la modalité épistémique et produisant une intersubjectivation empathique, tandis que l'étanchéité siège/tiers-actant s'ancre dans la modalité déontique et produit de l'intersubjectivation pragmatique. Les deux schèmes peuvent cependant se rapprocher, le savoir culturellement partagé étant aussi source de normes sociales.

L'expression des émotions se situe à l'articulation de plusieurs disciplines : si la linguistique s'intéresse aux stratégies linguistiques mobilisées et aux contraintes sémantiques et énonciatives qui les soustendent, certaines catégories jettent des ponts vers d'autres disciplines comme la psychologie, la neuropsychologie ou la sociologie. Il y a d'abord la sélection lexico-émotionnelle. L'expression allocentrée des émotions est réservée à quatre émotions : la peur, la joie, la tristesse et la honte, les trois premières étant des émotions de base et relevant du même schème d'intersubjectivation. Le statut d'émotion de base pourrait faciliter l'intersubjectivation et expliquer en partie les spécificités de l'expression de la honte. Les notions d'évaluation et d'intersubjectivation renvoient, pour la première, à la théorie de l'appraisal (voir Frijda 2007) et, pour la deuxième, aux questionnements récents sur les neurones miroirs (voir Gallese 2001). La distinction entre intersubjectivité empathique et intersubjectivité pragmatique permettrait de formuler des hypothèses concernant la configuration de l'opinion publique et des mouvements de masse (la fréquence des constructions allocentrées dans le discours du sport est une piste possible); l'analyse de discours peut apporter une contribution linguistique à de telles problématiques sociologiques.

\section{Références bibliographiques}

Aikhenvald, A. (2004). Evidentiality. Oxford : OUP.

Anscombre, J.-C. (2005). Temps, aspect et agentivité, dans le domaine des adjectifs psychologiques. Lidil, 32, [URL : http://lidil.revues.org/document110.html. Consulté le 25 janvier 2009.

Benveniste, E. (1966 [1960]). "Etre" et "avoir" dans leurs fonctions linguistiques. Problèmes de linguistique générale I. Paris : Gallimard, 187-207.

Bresson, D., Dobrovol'skij, D. (1995). Petite syntaxe de la 'peur'. Application au français et à l'allemand. Langue française, 105, 107-119.

Cadiot, (2007). Le morphème pour : polycatégorialité et unification sémantique. Cahiers de lexicologie, 90, 75-91.

Cislaru, G. (2008). L'intersubjectivation comme source de sens : expression et description de la peur dans les écrits de signalement. Les Carnets du Cediscor, 10, 117-136.

Culioli A. (1990). Pour une linguistique de l'énonciation : Opérations et représentations. Paris : Ophrys.

Dendale, (1994). Devoir épistémique, marqueur modal ou évidentiel ? Langue française, 102, 24-40.

Ekman, (1980). Biological and Cutural Contributions to Body and Facial Movement in the Expression of Emotions. In Rorty A. (ed.) Explaining Emotions. Los Angeles : California University Press.

Forest, R. (1999). Empathie et linguistique. Paris : PUF.

Forest, R. (2003). Empathie linguistique et point de vue. Cahiers de praxématique, 41, 85-104.

Frijda, N. H. (2007). The Laws of Emotion. Mahwah, NJ : Lawrence Erlbaum.

Gallese, V. (2001). The 'Shared Manifold' Hypothesis. From Mirror Neurons to Empathy. In Thompson E. (ed.) Between Ourselves. Second-person Issues in the Study of the Consciousness. Thorverton, UK - Charlottesille, USA : Imprint Academic, 31-50. 
Gibbard, A., 1996 [1990], Sagesse des choix, justesse des sentiments. Une théorie du jugement normatif, Paris, PUF.

Grandjean, D., Scherer, K.J. (2009). Théorie de l'évaluation cognitive et dynamique des processus émotionnels. In Sander D., Scherer K. (éds) Traité de psychologie des émotions. Paris : Dunod, 41-76.

Guentchéva, Z. (éd.) (1996). L'énonciation médiatisée. Louvain : Peeters.

Herslund, M. (2007). L'antipassif réfléchi en français et en danois. In Rousseau, A., Bottineau, D., Roulland, D. (éds) L'énoncé réfléchi. Rennes: PUR, 175-186.

Kay, (2003). Relativisme intra-locuteur. In Vandeloise C. (éd.) Langues et cognition. Paris : Lavoisier, 185-203.

Kerbrat-Orrechioni, C. (2000). Quelle place pour les émotions dans la linguistique du XXe siècle ? Remarques et aperçus. In Plantin C., Doury M., Traverso V. (éds) Les émotions dans les interactions, Lyon : PUL, 33-74.

Koselak, A. (2003). Approche sémantique du concept de honte. Pratiques, 117/118, 51-76.

Koselak, A. (2005). Quelle honte ! Ale wstyd! Observations sémantiques sur quelques emplois de honte et de wstyd. Roczniki Humanistyczne, LIII/5, 105-124.

Kreutz, Ph., 2001, « L'épidictique des émotions. In Dominicy M., Frédéric M. (éds) La mise en scène des valeurs. La rhétorique de l'éloge et du blâme, Lausanne, Delachaux et Niestlé, 107-134.

Kronning, H. (1996). Modalité, cognition et polysémie: sémantique du verbe modal devoir. Uppsala: Acta Universitatis Upsaliensis.

Kuno, S. (1987). Functional Syntax. Anaphora, Discourse and Empathy. Chicago - London: The University of Chicago Press.

Leeman, D. (1995). Pourquoi peut-on dire Max est en colère mais non *Max est en peur? Hypothèses sur la construction être en N. Langue française, 105, 55-69.

Le Querler, N. (1996). Typologie des modalités. Caen : PU de Caen.

Martin, F. (2003). Est-on honteux quand on est honteux pour autrui ? Les émotions normatives : analyse sémantique de la construction 'être $\psi$ pour NP humain'. In Colletta J.-M., Tcherkassof A. (éds) Les émotions. Cognition, langage et développement. Liège : Mardaga, 131-136.

Nuyts, J. (2001). Subjectivity as an evidential dimension in epistemic modal expressions. Journal of Pragmatic,s $33 / 3,383-400$

Rabatel, A. (1997). Une histoire du point de vue (Recherches textuelles 2). Université de Metz: diffusion Klincksieck.

Rimé, B. (2005). Le partage social des émotions. Paris : PUF.

Vion, R. (1992). La communication verbale. Analyse des interactions.Paris: Hachette.

Wierzbicka, A. (2006). English : Meaning and Culture. Oxord: Oxford UP.

Zahavi, D. (2001 [1996]). Husserl and Transcendental Intersubjectivity. A Response to the Linguistic-Pragmatic Critique. Athenes : Ohio University Press.

Zaliznjak, A. A., Shmelev, A. D. (2007). Sociativity, conjoining, reciprocity, and the Latin prefix com-. In Nedjalkov V.P. (ed.) Reciprocal Constructions I. Amsterdam - Philadelphia : John Benjamins, 209-229.

\footnotetext{
${ }^{1}$ Nos remerciements vont à Patrick Dendale, pour sa relecture, aux étudiants de L2 Lettres modernes et aux collègues du séminaire LMS (Modyco - CNRS/Paris 10), pour les discussions et échanges enrichissants.

${ }^{2}$ « [...] il peut être pertinent d'envisager l'attitude de l'auteur de productions langagières envers les participants des procès décrits par ce qu'il énonce, indépendamment de la description même des procès; attitude qui peut impliquer un certain degré d'identification à ces participants, ou du moins l'adoption de leur point de vue, sur les procès en question. » (Forest $1999: 16)$

${ }^{3}$ Par économie, nous assimilons ici le pronom sujet à N1. On trouvera dans Martin (2003) et Koselak (2003 et 2005) des réflexions concernant notamment l'expression de la honte par ce type de constructions.
} 
${ }^{4}$ Et cela au moins depuis l'Etique à Nicomaque d'Aristote. Les guillemets font allusion au débat, que nous n'ouvrirons pas ici, concernant le statut privé/individuel versus social des émotions.

${ }^{5}$ Sur cette dernière notion, nous renvoyons au travail de Rabatel (1997) et, pour une mise en regard des deux notions, à celui de Forest (2003).

${ }^{6}$ Les émotions de base sont considérées comme psychologiquement simples; elles apparaissent très tôt à priori sans impulsion sociale et tendent à être universelles. Il n'y a pas de consensus sur le nombre exact des émotions de base (quatre? sept ?), mais on y intègre généralement la peur, la joie, la tristesse, la colère.

${ }^{7}$ Contre au moins 100000 pour les quatre constructions retenues.

${ }^{8}$ Correspondant plutôt à la lecture b) du tableau 2 en 2.3 .

${ }^{9}$ En partant de la notion d'intériorité du procès, on peut se demander s'il n'y a pas une possibilité d'intériorisation du stimulus, en opposant je m'inquiète à cette situation m'inquiète et à j'inquiète mes grands-parents.

${ }^{10}$ D'autres formulations peuvent être proposées, comme Je suis content/heureux grâce/à cause de toi. Mais elles présentent l'inconvénient de neutraliser l'impact du stimulus. Les locuteurs interrogés (25 étudiants de L2 Lettres modernes) voient une différence du degré d'intentionnalité entre Je suis triste à cause de toi et Tu me rends triste.

$11 \mathrm{Si}$ l'on revient au cas de être fier, l'incongruité des structures causatives met en avant son écart par rapport aux quatre émotions retenues : ??Tu me rends fier et Je suis fier à cause de toi.

12 «Dans un cas, nous pouvons considérer l'ours comme le contenu de l'expérience de la peur; dans l'autre, comme le stimulus de cette expérience. Dans le premier cas, l'ours désigne un objet dans le monde ; dans le second, l'ours désigne un objet dans le monde des représentations mentales de Robert. » (Kay 2003 : 200)

${ }^{13}$ La notion d'évaluation renvoie à l'appraisal théory of emotion (pour une synthèse récente, voir Grandjean \& Scherer 2009). La dépendance des normes est développée par Gibbard (1996).

${ }^{14}$ On passe donc par une modalité appréciative.

${ }^{15} \mathrm{Au}$ sens de « nature joyeuse ». L'énoncé à la première personne a cependant un caractère artificiel...

16 Forest (1999: 89) souligne le lien entre empathie et évidentialité en donnant l'exemple de la narration d'événements passés dont l'énonciateur n'affirme pas avoir été le témoin, en oksapmin.

17 L'ensemble des stratégies de création de l'information mentionnées dans cette section rappelle les sources d'information telles qu'elles sont étudiées par les théories de l'évidentialité (Aikhenvald) ou de la médiation (Guentchéva) : perception, emprunt, inférence.

${ }^{18}$ On notera au passage que les constructions «être content/triste pour» sont particulièrement courantes dans le discours du et sur le sport. Cela semble confirmer l'effet particulièrement empathisant du sport, qui rassemble les masses en suscitant de la part du public une identification aux acteurs du sports.

${ }^{19}$ Kreutz (2001 : 124-133) compte l'admiration, le reproche, la fierté et la honte parmi les émotions épidictiques, dans le sens où elles peuvent être induites par des discours d'ordre épidictique, et insiste sur le lien structurel unissant le sujet des deux dernières émotions à l'agent de l'action.

${ }^{20}$ On peut signaler aussi les adverbes épistémiques tels que évidemment, probablement, etc., fonctionnant comme des marqueurs du positionnement épistémique du locuteur (Wierzbicka $2006:$ ch. 8).

${ }^{21}$ Deux remarques s'imposent ici : 1) la lecture subjective reste cependant possible, dans le sens où le sujet-locuteur peut s'appuyer sur des considérations personnelles non soumises à la validation par le sens ou le savoir commun ; 2) en suivant la typologie de Le Querler (1996), les constructions allocentrées articuleraient modalité intersubjective et modalité objective.

${ }^{22}$ On rappellera cependant l'ambivalence de la peur, qui semble se prêter assez facilement à une évaluation déontique (voir ci-dessus l'exemple de l'enfant timide).

23 Il nous semble cependant qu'une telle distinction n'est pas nécessaire a priori, même si l'on reconnaît généralement qu'il est possible de parler d'émotions sans en ressentir. Mais, en faisant la distinction en amont, l'auteure déplace le problème d'un cadre sémantique, où l'on peut s'interroger sur les possibilités offertes par les 
constructions allocentrées elles-mêmes, à un cadre vériconditionnel, où l'on s'interroge sur la réalité du vécu du sujet-siège, comme cela a été souligné en 1.1 .

${ }^{24}$ Ce constat rejoint en partie les conclusions de Martin (2003), pour qui les énoncés émotionnels en pour-objet ne sont pas empathiques, mais concernent des états méta-émotionnels. Cela se vérifie pour la honte, et reste une potentialité pour la peur. 